Research paper

\title{
A fluorescent multiplexed bead-based immunoassay (FMIA) for quantitation of IgG against Streptococcus pneumoniae, Haemophilus influenzae and Moraxella catarrhalis protein antigens
}

\author{
Dafne C. Andrade a,*, Igor C. Borges a , Hanna Laitinen ${ }^{\mathrm{b}}$, Nina Ekström ${ }^{\mathrm{b}}$, Peter V. Adrian ${ }^{\mathrm{c}}$, \\ Andreas Meinke ${ }^{\mathrm{d}}$, Aldina Barral $^{\mathrm{e}}$, Cristiana M. Nascimento-Carvalho ${ }^{\mathrm{f}}$, Helena Käyhty ${ }^{\mathrm{b}}$ \\ a Federal University of Bahia School of Medicine, Salvador, Bahia, Brazil \\ ${ }^{\mathrm{b}}$ National Institute for Health and Welfare, Helsinki, Finland \\ ${ }^{c}$ DST/NRF Vaccine Preventable Diseases, Respiratory and Meningeal Pathogens Research Unit, University of the Witwatersrand, Johannesburg, South Africa \\ d Valneva Austria, GmbH, Campus Vienna Biocenter 3, Vienna, Austria \\ e Pathology Department, Federal University of Bahia School of Medicine and Centro de Pesquisa Gonçalo Moniz, Fundação Oswaldo Cruz, Salvador, Bahia, Brazil \\ ${ }^{\mathrm{f}}$ Department of Pediatrics, Federal University of Bahia School of Medicine, Salvador, Bahia, Brazil
}

\section{A R T I C L E I N F O}

\section{Article history:}

Received 14 January 2014

Received in revised form 3 February 2014

Accepted 4 February 2014

Available online 11 February 2014

\section{Keywords:}

Antibody

Multiplex

Etiological diagnosis

Recombinant proteins

Serology

Validation

\begin{abstract}
A B S T R A C T
Streptococcus pneumoniae, Haemophilus influenzae, and Moraxella catarrhalis are pathogens commonly associated with infectious diseases in childhood. This study aimed to develop a fluorescent multiplexed bead-based immunoassay (FMIA) using recombinant proteins for the quantitation of serum IgG antibodies against these bacteria. Eight pneumococcal proteins (Ply, CbpA, PspA1, PspA2, PcpA, PhtD, SP1732-3 and SP2216-1), 3 proteins of $H$. influenzae (NTHi Protein D, NTHi0371-1, NTHi0830), and 5 proteins of $M$. catarrhalis (MC Omp CD, MC_RH4_2506, MC_RH4_1701, MC_RH4_3729-1, MC_RH4_4730) were used to develop the FMIA. Optimal coupling concentrations for each protein, comparison of singleplex and multiplex assays, specificity, reproducibility, and correlation to ELISA for six pneumococcal antigens were determined for validation. FMIA was then used to analyze acute and convalescent paired serum samples of 50 children with non-severe pneumonia. The coupling concentrations varied for different antigens, ranging from 1.6 to $32 \mu \mathrm{g}$ of protein/million beads. Correlation between singleplexed and multiplexed assays was excellent, with $\mathrm{R} \geq 0.987$. The FMIA was specific, reaching $>92 \%$ homologous inhibition for all specificities; heterologous inhibition $\geq 20 \%$ was found only in six cases. The assay was repeatable, with averages of intra-assay variation $\leq 10.5 \%$, day-to-day variation $\leq 9.7 \%$ and variation between technicians $\leq 9.1 \%$. Comparison with ELISA for pneumococcal antigens demonstrated good correlation with $\mathrm{R}$ ranging from 0.854 (PspA2) to 0.976 (PcpA). The samples from children showed a wide range of antibody concentrations and increases in convalescent samples. In conclusion, the FMIA was sensitive, specific, and repeatable, using small amounts of recombinant proteins and sera to detect
\end{abstract}

\footnotetext{
Abbreviations: AOM, acute otitis media; CAP, community-acquired pneumonia; CbpA, choline binding protein A; CV, coefficient of variation; EDC, 1-ethyl-3 (3-dimethylamino-propyl) carbodiimide-HCl; FMIA, fluorescent multiplexed bead-based immunoassay; MFI, Mean Fluorescence Intensity; NTHi, nontypeable $H$. influenzae; OMP CD, outer membrane protein CD; PBS, phosphate buffered saline; PcpA, pneumococcal choline binding protein A; PCV, pneumococcal conjugate vaccines; PhtD, pneumococcal histidine triad protein D; Ply, pneumolysin; PspA, pneumococcal surface protein A; R-PE, R-phycoerythrin; RT, room temperature; Sulfo-NHS, Hydroxysulfosuccinimide

* Corresponding author at: Praça XV de Novembro, s/n - Largo do Terreiro de Jesus, Salvador, Bahia CEP 40025-010, Brazil. Tel.: + 557132835568 ; fax: + 5571 32835567.

E-mail address: andradedafne@yahoo.com.br (D.C. Andrade).
} 
antibodies against S. pneumoniae, H. influenzae and M. catarrhalis. The methodology would be suitable for studies investigating etiological diagnosis and in experimental vaccine studies.

(c) 2014 Elsevier B.V. All rights reserved.

\section{Introduction}

Streptococcus pneumoniae, Haemophilus influenzae, and Moraxella catarrhalis are pathogens commonly associated with respiratory tract infections in childhood, such as community-acquired pneumonia (CAP) and acute otitis media (AOM) (Casey et al., 2010; Rudan et al., 2008). Nevertheless, the accurate etiologic diagnosis of infection by these agents is difficult, and most serological tests can only detect a limited number of strains of each pathogen, reinforcing the need for new assays that recognize the immune response to these bacteria regardless of, for example, the serotypes or variations in other surface structures. Also, as no licensed vaccines have been introduced for nontypeable $H$. influenzae (NTHi) and $M$. catarrhalis, and the ones available against S. pneumoniae do not provide coverage against all serotypes (Rodgers and Klugman, 2011), new antigens have been increasingly studied as vaccine candidates. Therefore, the development of serological assays for these pathogens based on specific and conserved antigens for each species is warranted.

Several pneumococcal protein antigens have been well characterized and investigated for the development of new vaccines (Principi and Esposito, 2011) and have also been increasingly used in serological assays. Among them, pneumolysin (Ply), choline binding protein $A(C b p A)$, pneumococcal surface protein A (PspA), pneumococcal choline binding protein $A$ (PcpA) and pneumococcal histidine triad protein $\mathrm{D}$ (PhtD) are promising antigens for both purposes. Ply is a highly conserved cytotoxin released during autolysis that interacts in many ways with the host immune system (Tai, 2006; van der Poll and Opal, 2009). CbpA (Brooks-Walter et al., 1999; Tai, 2006; van der Poll and Opal, 2009) and PspA (Briles et al., 2000; Crain et al., 1990; Croney et al., 2012; Tai, 2006; van der Poll and Opal, 2009) are also present in most strains of S. pneumoniae and play several roles in the pathogenesis of pneumococcal infection. PcpA is a choline binding protein present in the majority of $S$. pneumoniae virulent strains involved in adherence to epithelial cells (Khan et al., 2012). Similarly, PhtD is a highly conserved surface protein from the Pht family (Adamou et al., 2001), that has been recently recognized as an adhesin (Khan and Pichichero, 2012).

Among the protein antigens of $H$. influenzae, protein $\mathrm{D}$ is an important vaccine candidate, as it is a conserved virulence factor present in all strains (Poolman et al., 2000). Further, it has been reported that protein $D$ as a carrier in pneumococcal conjugate vaccine (PCV) provides protection against AOM caused by $H$. influenzae (De Wals et al., 2009). Several adhesins have also been identified in $M$. catarrhalis that would be suitable as diagnostic targets or as vaccine candidates, such as the outer membrane protein CD (OMP CD), an adhesin that also has other functions in pathogenesis, and Msp22, a surface lipoprotein (Murphy and Parameswaran, 2009; Saito et al., 2013; Smidt et al., 2013). Although there is no currently licensed vaccine using all the aforementioned proteins (except for the use of protein $\mathrm{D}$ as a carrier in one of the PCVs), these antigens merit further investigation.
To date, the quantitation of antibodies to these pathogens relies mostly on the use of ELISA, which is recognized as a specific and sensitive technique (Korppi et al., 2008). Nevertheless, when the evaluation of the immune response to multiple antigens is required, ELISA becomes considerably time-consuming and expensive. Additionally, the performance of one test per analyte demands a large volume of serum, which is usually hard to obtain when dealing with pediatric patients. In this setting, the use of a multiplexed assay allows the detection of antibodies against several antigens simultaneously with high sample throughput and reduction in the amount of serum needed, which represent important advantages when performing seroepidemiological studies or experimental vaccine trials. In this context, we describe in detail the development and validation of a multiplexed immunoassay using Luminex xMAP® Technology with recombinant proteins for the detection and quantitation of antibodies directed to S. pneumoniae, $H$. influenzae, and M. catarrhalis.

\section{Materials and methods}

\subsection{Reagents}

The presence of antibodies against S. pneumoniae was investigated using eight distinct recombinant pneumococcal protein antigens: Ply, CbpA, PspA family 1 (PspA1), PspA family 2 (PspA2), PcpA, PhtD, SP1732-3, and SP2216-1. Antibodies directed to $H$. influenzae were assayed using the recombinant proteins NTHi Protein D, NTHi0371-1, and NTHi0830. NTHi0371-1 is a fragment of the protein heme/ hemopexin utilization protein A (gene hxuA) representing the first domain of the mature protein which includes the hemagglutinin activity domain. The protein NTHI0830 is the outer membrane antigenic lipoprotein B. M. catarrhalis antibodies were investigated using the recombinant protein antigens MC Omp CD, MC_RH4_2506, MC_RH4_1701, MC_RH4_3729-1, and MC_RH4_4730 (Smidt et al., 2013). The combination of antigens was chosen with the purpose of providing a good coverage of the bacterial etiological agents causing respiratory tract infections and based on the information available on the immune response to antigens from these pathogens.

For the development of this fluorescent multiplex beadbased immunoassay (FMIA), a truncated PcpA (Posfay-Barbe et al., 2011) and PhtD (Seiberling et al., 2012) were kindly supplied by Sanofi Pasteur (Sanofi Pasteur S.A., Marcy L'Etoile, France); SP1732-3 and SP2216-1 (Giefing et al., 2008), NTHi Protein D, NTHi0371-1, NTHi0830, MC Omp CD, MC_RH4_2506, MC_RH4_1701, MC_RH4_3729-1, and MC_RH4_4730 were from Andreas Meinke (Valneva, Vienna, Austria). The antigens Ply (Ogunniyi et al., 2001), CbpA (Orihuela et al., 2009), and PspA1 (UAB055) (Brooks-Walter et al., 1999; Shaper et al., 2004) were kindly supplied by Elaine Tuomanen (St. Jude Children's Research Hospital, Memphis, TN, USA) and PspA2 (UAB099) 
(McDaniel et al., 1994) by the group at University of Alabama in Birmingham (Birmingham, AL, USA; Susan Hollingshead, David Briles, and Pat Coan).

Comparison with ELISA was performed using samples previously analyzed by this technique with the antigens Ply, CbpA, PspA1, PspA2, PcpA, and PhtD. In particular, the Ply protein previously used in ELISA had been supplied by Sanofi-Pasteur (Lyon, France), and the antigens CbpA and PhtD had been supplied by GlaxoSmithKline (Rixensart, Belgium); the remaining antigens were provided by the same suppliers as the ones for the FMIA.

Hydroxysulfosuccinimide (Sulfo-NHS) and 1-ethyl-3 (3dimethylamino-propyl) carbodiimide- $\mathrm{HCl}$ (EDC) were obtained from Thermo Fisher Scientific, Rockford, IL, USA. R-phycoerythrin (R-PE)-conjugated AffiniPure goat antihuman IgG, $\mathrm{Fc}_{\gamma}$ Fragment specific was obtained from Jackson ImmunoResearch Laboratories Inc. (Westgrove, PA, USA). Carboxylated microspheres were obtained from Luminex Corporation, Austin, TX, USA.

\subsection{Serum samples}

An immunoglobulin preparation (Venogamma, Finnish Red Cross, Helsinki, Finland) was used to create dilution curves, and a solution of phosphate buffered saline (PBS) containing 10\% Fetal Bovine Serum (F-PBS) was used as blank and as the analysis buffer for the dilution of samples and controls. Patient sera that had been sent to the National Institute for Health and Welfare (Finland) for antibody testing were used without identification to test the beads conjugated with pneumococcal antigens. Sera from patients with AOM from the FinOM cohort study (Kilpi et al., 2001) were used for setting up and validation of $H$. influenzae and $M$. catarrhalis assays. Twenty-two in-house serum samples that had been analyzed for antibodies against pneumococcal protein antigens by ELISA previously were used for comparison of ELISA and FMIA. Twenty-two samples from Brazilian children with non-severe pneumonia were used to assess inter-assay variation. Furthermore, the developed assay was finally used to analyze the antibody concentrations to different antigens in paired samples from 50 Brazilian children aged 2-59 months with non-severe CAP.

The use of the samples was approved by the Ethics Committee from the Federal University of Bahia in Brazil, and the Ethics Committees from the National Institute for Health and Welfare (former National Public Health Institute), Tampere Health Center and Tampere University Hospital in Finland.

\subsection{Coupling of proteins to the beads}

The investigation of the presence of antibodies to $S$. pneumoniae, $H$. influenzae, and $M$. catarrhalis was performed using a bead based assay (XMAP® Technology, Luminex Corporation) in which the recombinant proteins were conjugated to magnetic fluorescent microspheres using a 2-step carbodiimide reaction (Pickering et al., 2002a). Beads from bead regions not directly adjacent to each other were conjugated with the aforementioned antigens to develop a nine-plex assay: Ply, CbpA, PcpA, PhtD, SP1732-3, and SP2216-1 were conjugated in one bead region each. PspA1 and PspA2, which represent the vast majority of PspA identified on pneumococcal isolates and share a high level of homology (Briles et al., 2000; Tai, 2006), were conjugated in the same bead region. All $H$. influenzae and all M. catarrhalis proteins were also conjugated in one bead region per bacterium. Prior to conjugation, the proteins stored in buffers containing amino-acids were individually dialyzed using the appropriate dialyzing cassettes (Slide-A-Lyzer® G2 Dialysis Cassette, Thermo Fisher Scientific, Rockford, IL, USA) to prevent the coupling of the beads with any other component besides the desired antigen.

After adequate homogenization, $6.25 \times 10^{6}$ beads were transferred to low-binding tubes and centrifuged for $4 \mathrm{~min}$ at $9650 \times \mathrm{g}$ at room temperature. The supernatant was then discarded and the beads were activated by addition of $1 \mathrm{ml}$ of PBS at pH 7.3 containing $2.5 \mathrm{mg}$ of EDC and $2.5 \mathrm{mg}$ of Sulfo-NHS (final $\mathrm{pH}=6.1$ ) (Lal et al., 2005) and incubated for $20 \mathrm{~min}$ in the dark, at room temperature (RT), under constant rotation at $600 \mathrm{rpm}$. The activated microspheres were washed 2 times with PBS ( $\mathrm{pH} 7.3$ ) by centrifugation at $9650 \times \mathrm{g}$ for $4 \mathrm{~min}$ and resuspended with $1 \mathrm{ml}$ of the diluted protein solution in PBS at $\mathrm{pH}$ 7.3. The beads were then incubated for $90 \mathrm{~min}$, in the dark, at RT, rotating at $600 \mathrm{rpm}$. Subsequently, the protein coupled microspheres were washed 2 times as described above and resuspended in $1 \mathrm{ml} \mathrm{F-PBS}$ containing $0.01 \%$ of sodium azide. After homogenization, the beads were counted in a dilution of $1 / 100$ in PBS and then stored at $4{ }^{\circ} \mathrm{C}$ in the dark. The storage buffer for Ply coupled microspheres was F-PBS containing 10\% glycerol and $0.01 \%$ sodium azide. Ply coupled microspheres were stored at $-20{ }^{\circ} \mathrm{C}$.

For uncoupled beads, the described procedure was followed, but only PBS, instead of the diluted antigen solution, was added.

\subsection{Antibody assay}

The samples and Venogamma were serially diluted $1 / 4$ in F-PBS, starting from a 1/100 dilution. True duplicates were used throughout the development of the protocol and the Mean Fluorescence Intensity (MFI) values for each dilution, reflecting the antibody levels, were averaged. The beads were diluted in order to generate a working concentration of 1750 beads per bead region per well. A 96 well plate (Costar 3915, Thermo Fisher Scientific, Rockford, IL, USA) was used to perform the assay. Initially, $25 \mu \mathrm{l}$ of diluted Venogamma or serum and $25 \mu$ of the bead dilution were transferred to the wells. The plate was incubated on a shaker at $900 \mathrm{rpm}$ for $1 \mathrm{~min}$ and $600 \mathrm{rpm}$ for $60 \mathrm{~min}$ at room temperature in the dark. After incubation, the plate was washed twice with PBS with $0.05 \%$ Tween using a Biotek ELx405 Select Deep Well Microplate Washer (BioTek Instruments, Inc., VT). To each well, $50 \mu$ of a 1/100 dilution of R-PE conjugated anti-human IgG in PBS was added and then the plate was incubated at $900 \mathrm{rpm}$ for $1 \mathrm{~min}$ and $600 \mathrm{rpm}$ for $30 \mathrm{~min}$ at room temperature. The plate was then washed again as above, and $80 \mu \mathrm{l} /$ well of PBS was added. The plate was again incubated at $600 \mathrm{rpm}$ for $5 \mathrm{~min}$. Measurements were made using a MAGPIX device in combination with XPONENT software (XMAP® Technology, Luminex Corporation, Austin, TX, USA).

The ELISA was performed as previously described (Simell et al., 2008). 


\subsection{Determination of the optimal protein concentration for conjugation}

Beads conjugated with different protein concentrations were tested with the above protocol to assess the optimal concentration for each antigen. Serial 1/4 dilutions of Venogamma, starting at $1 / 100$, were used to create the dilution curve by plotting the log MFI against the dilution. The MFI values obtained for 8 serum samples were also used to evaluate the comparability between the beads conjugated with different concentrations of the same antigen (data not shown). The decision on final concentration of each antigen was based on the width of the dynamic range it produced. Testing of samples from patients was performed to evaluate the pattern of variation of fluorescence readings generated by the conjugated beads.

For the pneumococcal antigens, the tested concentrations included $1.6,8,16,32,80$, and $160 \mu \mathrm{g} /$ million beads. Based on the results of the initial tests, for the $H$. influenzae and $M$. catarrhalis antigens were tested at concentrations 1.6, 8, and $16 \mu \mathrm{g}$ of each protein/million beads.

\subsection{Comparison of singleplex and multiplex assays}

The MFI values obtained from each bead set tested in singleplex and in multiplex format were used to evaluate the comparability between the assays. The correlation coefficients $(r)$ were calculated using log based data.

\subsection{Linearity}

To determine the linear range of the dilution curve, and to find optimal dilutions of the sera to be used when studying patient samples, five samples with known varying levels of antibodies to the different antigens were sequentially diluted $1 / 2$, starting from $1 / 100$ up to $1 / 12,800$. Data from serial $1 / 4$ dilutions of Venogamma starting at $1 / 100$ were also used.

\subsection{Specificity}

Inhibition-of-binding studies were conducted with samples diluted $1 / 100$ and the homologous antigen was added to its respective aliquot to reach a concentration of $24 \mu \mathrm{g}$ protein/million beads. For the evaluation of the bead sets conjugated to PspA1 and 2, $H$. influenzae antigens and $M$. catarrhalis antigens, all the proteins of each bead set were added simultaneously to its respective well, at a concentration of $24 \mu \mathrm{g}$ of each protein/million beads. A 1/100 dilution of each serum, with no added antigen, was used as a control. The prepared sample dilutions were stored for $12 \mathrm{~h}$ at $4{ }^{\circ} \mathrm{C}$ and then assayed in multiplexed format, as described above. The test was performed in three separate assays, with a total of three sera tested with the addition of pneumococcal proteins, three sera with $H$. influenzae proteins, and three sera with $M$. catarrhalis proteins. All the used sera had high MFI values, reflecting high concentrations of antibodies, against most of the studied antigens: 1079 for Ply, 3592 for CbpA, 1718 for PspA1 and 2, 1443 for PcpA, 5783 for PhtD, 7896 for SP1732-3, 6937 for SP2216-1, 1296 for H. influenzae antigens and 2932 for $M$. catarrhalis antigens. Homologous and heterologous inhibition was calculated as the percentage of reduction of the MFI values for each inhibitor compared to the control wells with no added inhibitor. To assess unspecific binding of IgG to the beads, uncoupled beads were used in the assay with a serum sample with known high MFI values for the studied antigens.

\subsection{Comparison with ELISA}

Comparison of ELISA and FMIA was done by using 22 in house serum samples that had been analyzed by ELISA previously for anti-pneumococcal antibodies against Ply, CbpA, PspA1, PspA2, PcpA, and PhtD, and correlation coefficients $(r)$ were calculated using log based data. The results are given as log MFI for the FMIA and, in ELISA, as the log of the antibody concentration in $\mu \mathrm{g} / \mathrm{ml}$ for CbpA, PspA1, PspA2 and PhtD, and of ELISA units for Ply and PcpA.

\subsection{Repeatability}

Repeatability of the FMIA was assessed by determining both intra-assay and inter-assay variation. Intra-assay variation was calculated from 16 repetitions in the same plate of five different serum samples using the multiplex assay. The variation on different strata of MFI values was also evaluated, considering the mean of the 16 repetitions of each sample for each bead as one value to be stratified on the following categories of MFI values: $<500 ; \geq 500$ and $<1000 ; \geq 1000$ and $<5000$; $\geq 5000$ and $<10,000$; and $\geq 10,000$.

Inter-assay variation was calculated from the results of three repetitions in multiplex on different days of 22 samples of Brazilian children with non-severe CAP. The variation between two different technicians was also evaluated using the same set of serum samples. The percentage coefficient of variation (CV) was calculated between each of these results and averaged.

The repeatability of the results generated by different batches of conjugated beads was evaluated using serial dilutions of Venogamma in distinct singleplex assays. The results obtained using the distinct batches were compared by calculating the correlation coefficients $(r)$ using log based data.

\subsection{Quantitation of IgG in sera of children with CAP}

As a preliminary test to verify the function of the assay in a practical setting for etiologic purposes, the FMIA was used to quantitate the antibodies against S. pneumoniae, $H$. influenzae, and M. catarrhalis in paired serum samples from 50 children with non-severe CAP, evaluating the differences in antibody concentration between the acute and convalescent samples collected from 2 to 4 weeks apart. Data on the age from these children at admission were averaged.

\section{Results}

\subsection{Determination of the optimal protein concentration for conjugation}

We determined the optimal protein concentration by performing assays including the dilution curves for Venogamma and by determining the MFI values for eight serum samples with varying antibody concentrations. The maximum MFI values varied from approximately 2000 up to 15,000 depending 
on the evaluated bead set. In the first experiments it was noticed that 80 and $160 \mu \mathrm{g} / \mathrm{million}$ beads did not give curves differing from beads coupled with $32 \mu \mathrm{g} /$ million beads. Fig. 1 . shows the data for the dilution curve for Venogamma and for the MFI values of the serum samples for beads coupled with Ply, PspA1, and PspA2, as examples. After having obtained the pneumococcal protein data, the optimal concentrations for the antigens from $H$. influenzae and $M$. catarrhalis were determined, using all antigens at the same concentrations (1.6, 8, and $16 \mu \mathrm{g}$ of each antigen/million beads).

These tests demonstrated that in most cases lower protein concentrations could reach MFI values as high as the ones with beads conjugated with higher concentrations, so we could safely use the more economic option. Ply and PspA1 had their optimal concentration defined at $16 \mu \mathrm{g} /$ million beads; PcpA at $32 \mu \mathrm{g} /$ million beads; and CbpA, PspA2, PhtD, SP1732-3, and SP2216-1 at $1.6 \mu \mathrm{g} /$ million beads. The proteins from $H$. influenzae and $M$. catarrhalis had their optimal concentration defined at $8 \mu \mathrm{g} / \mathrm{million}$ beads.

\subsection{Comparison of singleplex and multiplex assays}

The comparison of the MFI values obtained with the singleplex and with the multiplex assays is shown in Fig. 2.
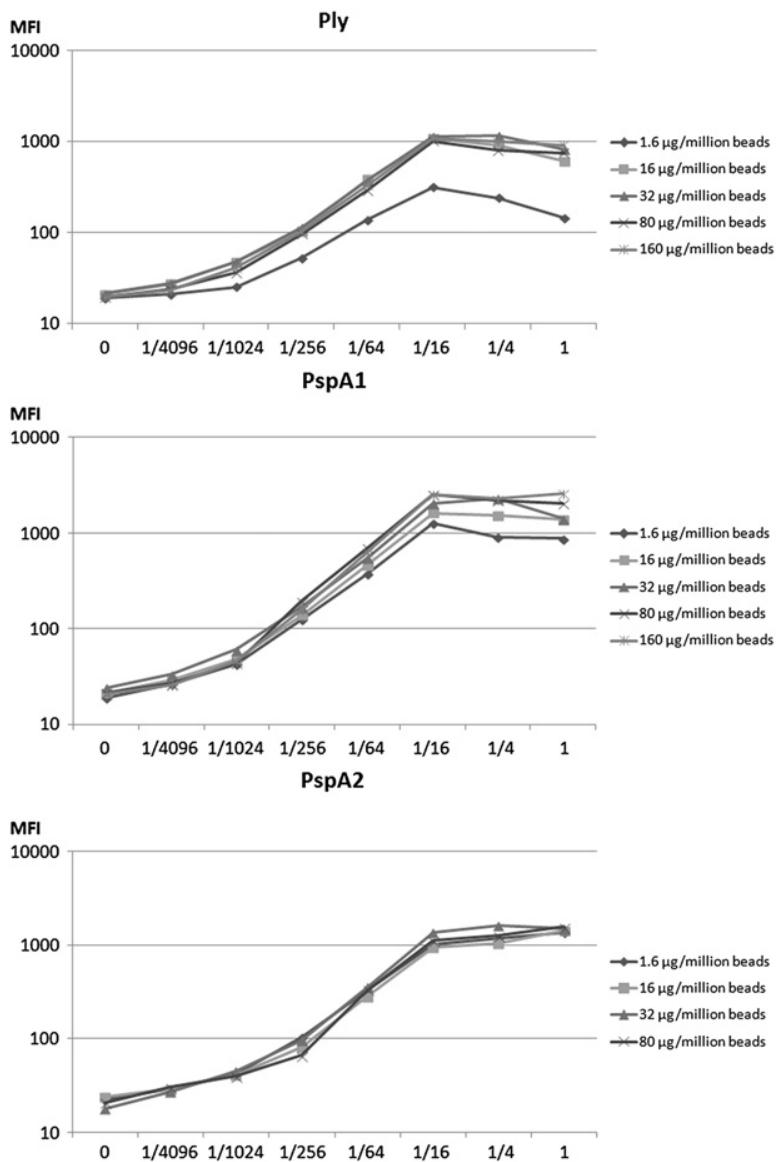

Fig. 1. Mean Fluorescence Intensity (MFI) of serial dilutions of Venogamma plotted against the dilution factor assayed with beads coupled with Ply, PspA1 and PspA2 in different protein concentrations as examples.
The fluorescent signals generated in singleplex assays were practically identical to the ones in multiplex assays for all bead sets.

\subsection{Linearity}

Data from the serial dilutions of both Venogamma and the serum samples were used to define the linear range of the assay for each bead set. Fig. 3.a depicts the dilution curves using seven serial 1/4 dilutions from Venogamma for all the bead sets conjugated on the final protein concentrations defined by the previously described titration. This figure shows that distinct antigens had different ranges of the linear phase of the dilution curve. For instance, the bead sets conjugated with CbpA, PhtD, SP1732-3, and SP2216-1 had similar parallel slopes that were linear over a 64-fold dilution range. The bead sets conjugated with Ply, PspA1 and 2, PсpA, $H$. influenzae proteins, and $M$. catarrhalis proteins, on the other hand, exhibited low MFI values when evaluated using Venogamma. Nevertheless, when these antigens were evaluated using the dilution curves generated using 5 serum samples with varying antibody levels (depicted in Fig. 3.b. as an example), they demonstrated a linear range over a 32- to 64 -fold dilution range. Based on the aforementioned data, the dilutions $1 / 400$ and $1 / 1600$ were considered to provide MFI values which usually represented the linear range for all the bead sets tested. For samples with high antibody concentrations with MFI values outside the linear range, further dilutions were performed.

\subsection{Specificity}

Homologous inhibition was accomplished with all protein antigens (mean inhibition of $96.3 \pm 2.8 \%$ ), successfully neutralizing the binding between the proteins conjugated to the beads and the antibodies against these antigens. The mean homologous inhibition for each bead set was: 92.8\% for Ply (range: $85-97 \%$ ); 97.3\% for CbpA (range: 93-99\%); 93.4\% for PspA1 and 2 (range: 91-97\%); 96.8\% for PcpA (range: 96-98\%); 99\% for PhtD (range: 98-100\%); 99.4\% for SP1732-3 (range: 99-100\%); $99.2 \%$ for SP2216-1 (range: 99-100\%); $96.5 \%$ for $H$. influenzae antigens (range: 95-97\%); and 92.2\% for M. catarrhalis antigens (range: 83-97\%). Inhibition of binding by heterologous protein antigens was detected only in few cases. The addition of $\mathrm{CbpA}$ resulted in a reduction of the MFI values for Ply (25.2\%), PspA1 and 2 (26.9\%), H. influenzae antigens (22.2\%), and $M$. catarrhalis antigens (21.9\%). Heterologous inhibition was also detected for PspA1 and 2 (26.2\%) and for the $H$. influenzae beads (30.8\%) when $M$. catarrhalis antigens were added.

Low MFI values $(<20)$ were obtained when uncoupled beads were used in the assay for a serum sample with known high antibody titers.

\subsection{Comparison with ELISA}

The comparison of the results from ELISA and the FMIA is depicted in Fig. 4. Good correlation was found, with the correlation coefficients ranging from 0.854 for PspA2 to 0.976 for PcpA. 
Ply
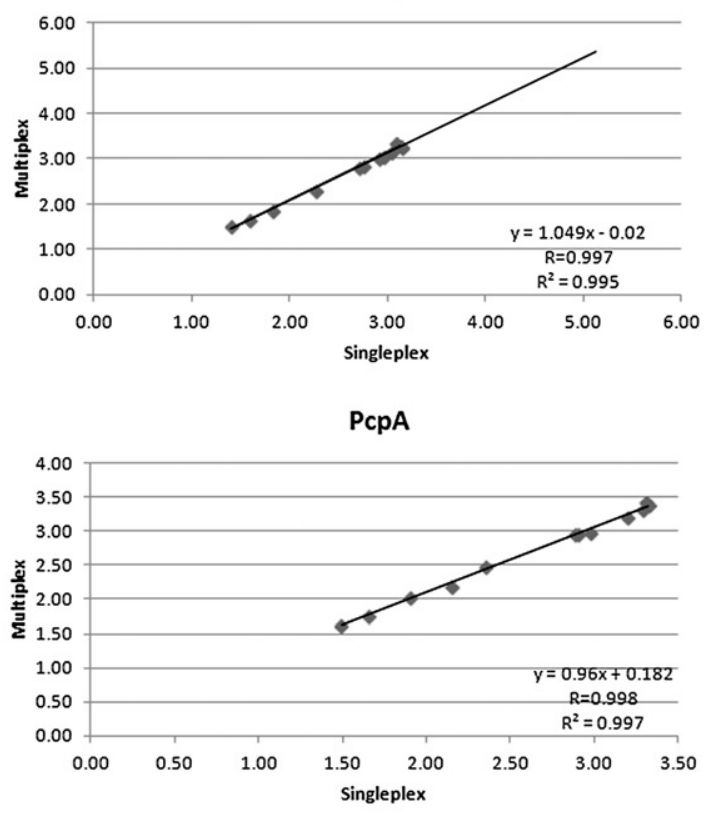

SP2216-1

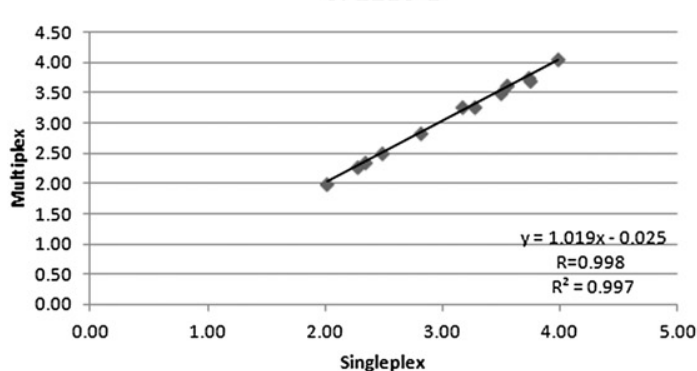

CbpA

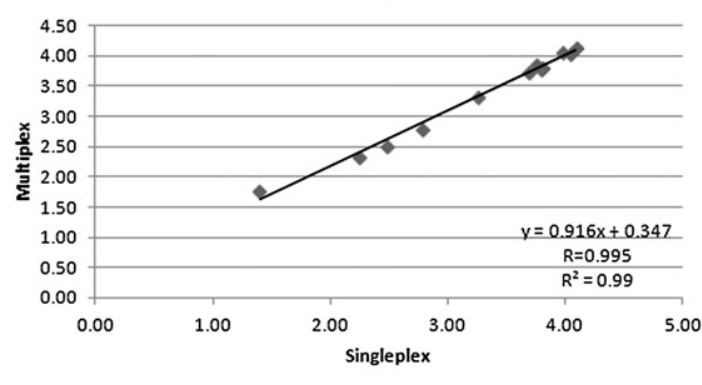

PhtD

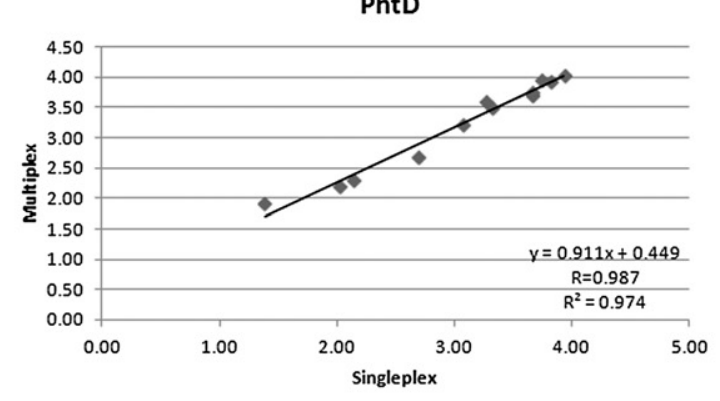

H. influenzae

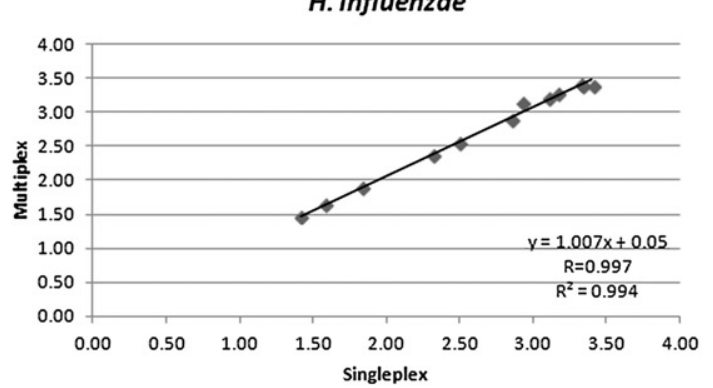

PspA 1 and 2

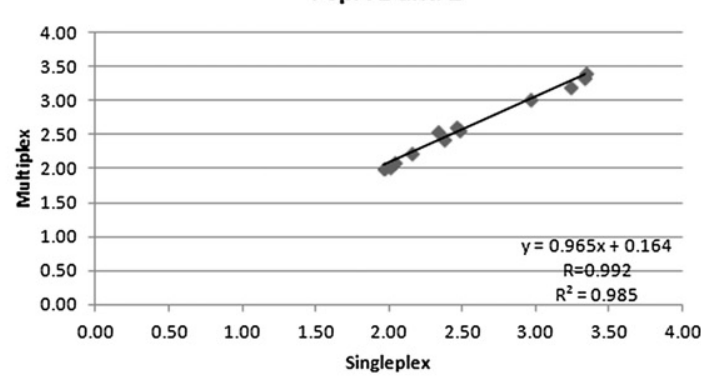

SP1732-3

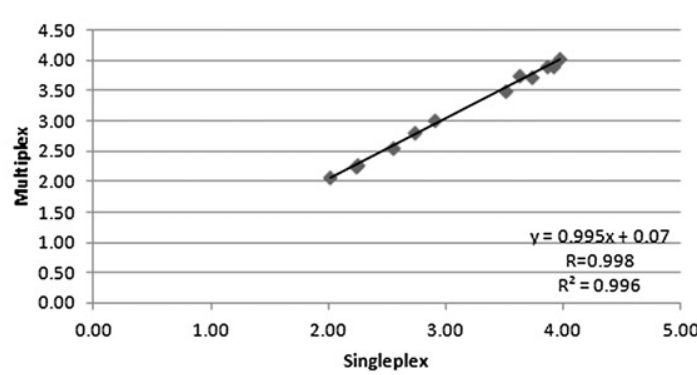

M. catarrhalis

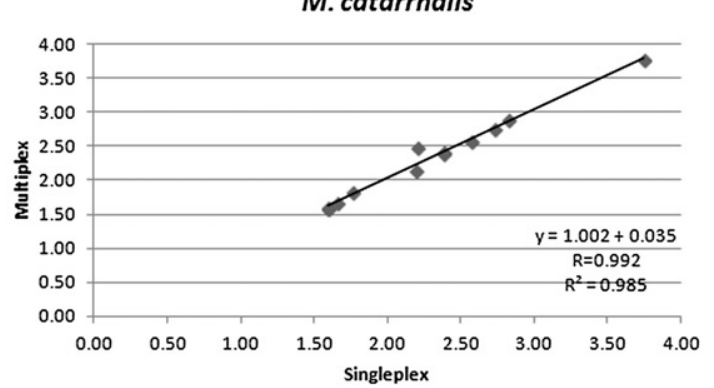

Fig. 2. Comparison of the Mean Fluorescence Intensity (MFI) values obtained in singleplex and multiplex assays after log transformation $(\mathrm{N}=12)$. 
a

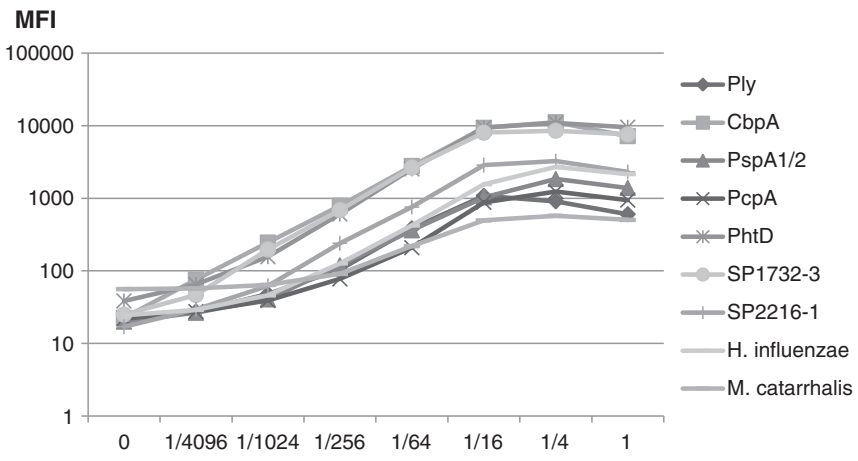

b

MFI

Ply
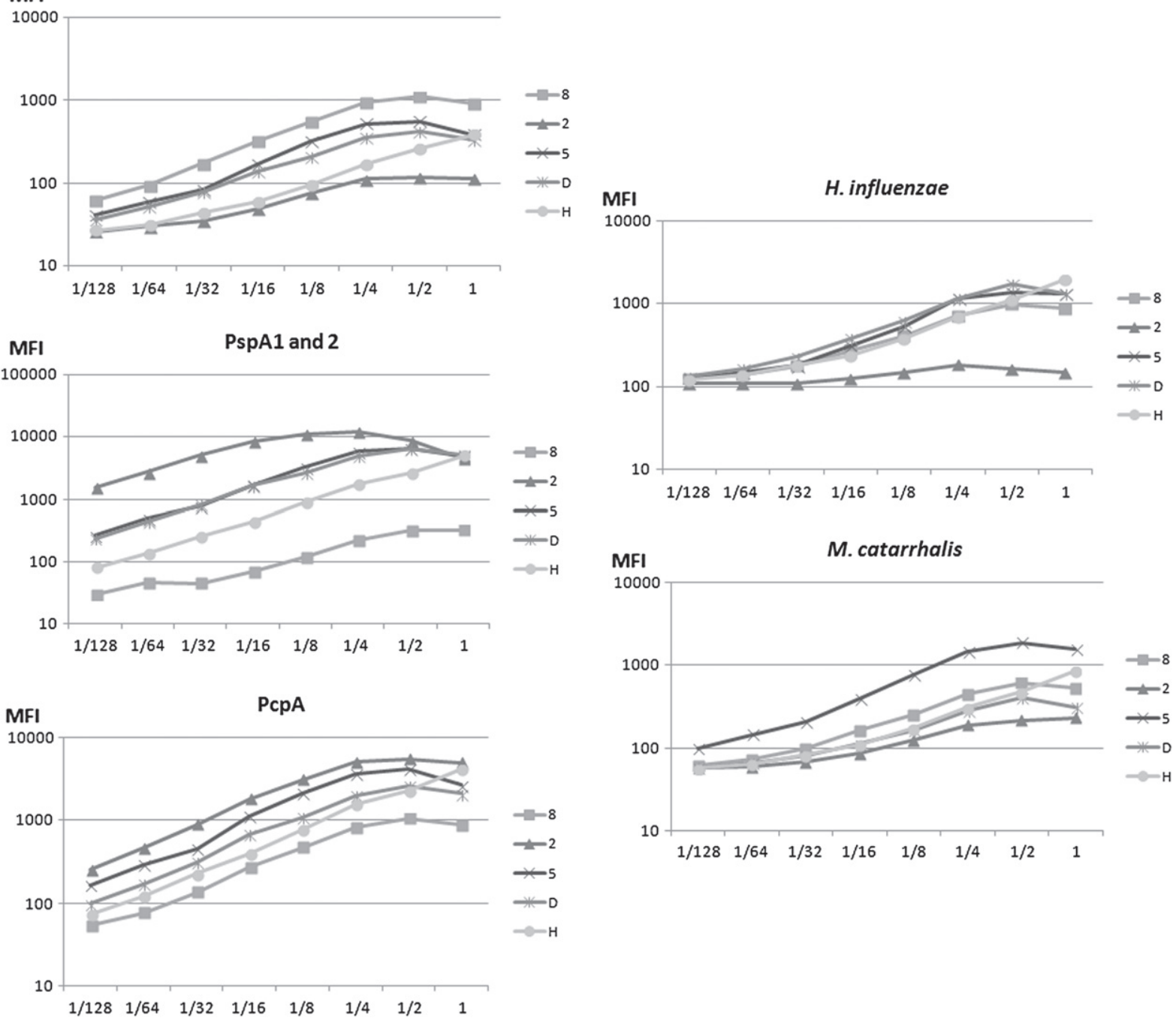

$\begin{array}{llllllll}1 / 128 & 1 / 64 & 1 / 32 & 1 / 16 & 1 / 8 & 1 / 4 & 1 / 2 & 1\end{array}$

Fig. 3. a. Mean Fluorescence Intensity (MFI) of serial dilutions of Venogamma assayed with 9 sets of beads coupled with protein antigens plotted against the dilution of the serum. b. Dilution curves of 5 samples for the bead sets conjugated with Ply, PspA1 and 2, PcpA, H. influenzae antigens and M. catarrhalis antigens plotted against the dilution as examples. The samples were named randomly, with numbers representing serum samples sent to the National Institute for Health and Welfare (Finland) for antibody testing and letters representing infant samples from the FINOM study. 


\subsection{Repeatability}

\subsubsection{Intra-assay variation}

Variation for the assays repeated 16 times on the same plate was low for all tested bead sets. The mean CV was $8.2 \%$ for Ply (range 6-12), 8.2\% for CbpA (range 2-13), 6.3\% for PspA1 and PspA2 (range 2-10), 6.8\% for PcpA (range 3-11), $6.9 \%$ for PhtD (range $3-10$ ), 8.5\% for SP1732-3 (range 4-12), 7.7\% for SP2216-1 (range 4-11), $10.5 \%$ for $H$. influenzae antigens (range 6-14), and $8.6 \%$ for $M$. catarrhalis antigens (range 6-12). The variation according to different strata of MFI values is shown in Table 1.

\subsubsection{Inter-assay variation}

The CV of the MFI values obtained from 3 repetitions of 22 samples on different days was 7.9\% for Ply, 7\% for CbpA, 7.9\% for PspA1 and PspA2, 7.9\% for PcpA, 7.9\% for PhtD, 6.7\% for SP1732-3, 6.6\% for SP2216-1, 8.6\% for $H$. influenzae antigens
Table 1

Intra-assay variation of different strata of Mean Fluorescence Intensity (MFI) values. Sixteen repetitions were performed for each of the 5 samples and assayed using the 9 bead sets. The values given are the coefficient of variation (CV\%) calculated for each sample/bead set.

\begin{tabular}{lcl}
\hline Mean MFI value & $\begin{array}{l}\text { Number of values } \\
\text { considered }\end{array}$ & $\begin{array}{l}\text { Mean variation } \pm \text { standard } \\
\text { deviation }(\%)\end{array}$ \\
\hline$<500$ & 5 & $8.62 \pm 2.53$ \\
$500 \geq$ and $<1000$ & 13 & $10.6 \pm 2.97$ \\
$1000 \geq$ and $<5000$ & 16 & $8.56 \pm 2.87$ \\
$5000 \geq$ and $<10,000$ & 7 & $6.29 \pm 2.98$ \\
$\geq 10,000$ & 4 & $3.25 \pm 1.5$ \\
\hline
\end{tabular}

and $9.7 \%$ for $M$. catarrhalis antigens. The variation between technicians was also low: the CV of the MFI values was $6.2 \%$ for Ply, 6.6\% for CbpA, 5.6\% for PspA1 and PspA2, 6.4\% for PcpA, 9.1\% for PhtD, 5\% for SP1732-3, 5.6\% for SP2216-1, 8.6\% for $H$. influenzae antigens, and $7 \%$ for $M$. catarrhalis antigens.
Ply

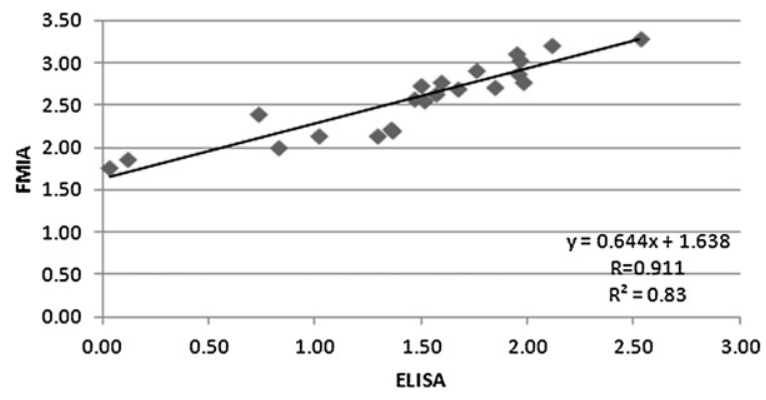

PspA 1

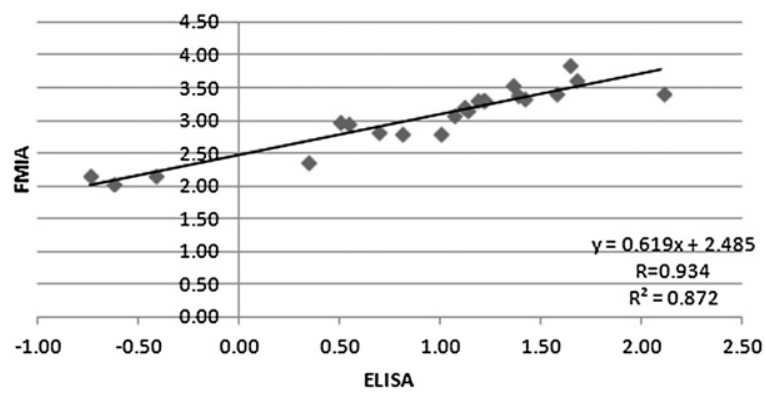

PcpA

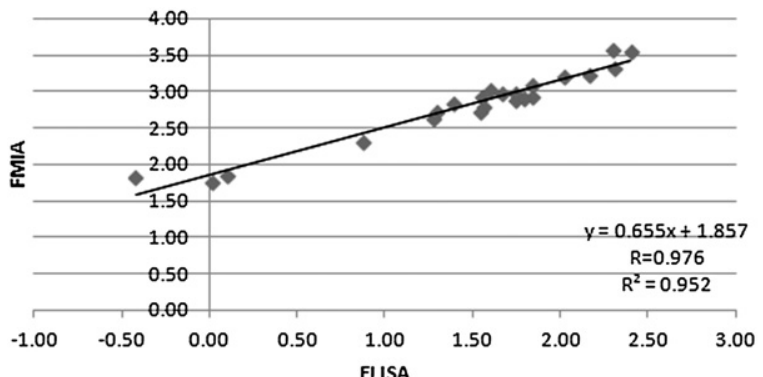

CbpA

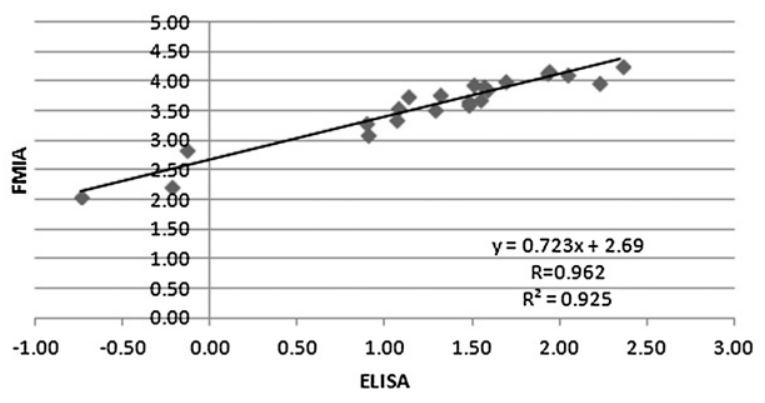

PspA2

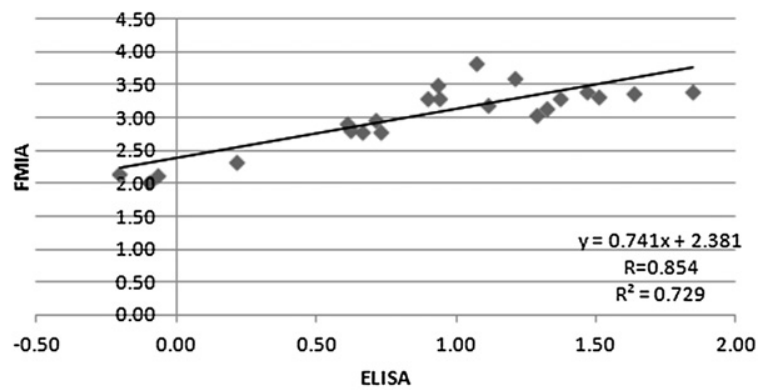

PhtD

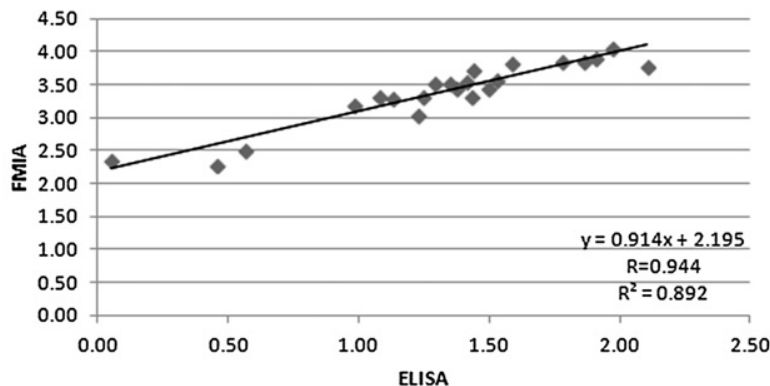

Fig. 4. Correlation between the results with ELISA and the Mean Fluorescence Intensity (MFI) values obtained with the FMIA for Ply, PspA1 and 2, CbpA, PcpA and PhtD after log transformation. PspA1 and 2 were analyzed separately on ELISA and on the same bead set on the FMIA. The results were given as log MFI for the FMIA and, in ELISA, as the log of the antibody concentration in $\mu \mathrm{g} / \mathrm{ml}$ for CbpA, PspA1, PspA2 and PhtD and of ELISA units for Ply and PcpA ( $\mathrm{N}=22$ ). 


\subsubsection{Repeatability of the coupling reaction}

The comparison of the results of serial dilutions of Venogamma analyzed with different batches of conjugated beads for all studied antigens is depicted in Fig. 5. A good correlation was demonstrated for all bead sets, with a correlation coefficient $\geq 0.991$.

\subsection{Quantitation of $\operatorname{Ig} G$ in sera of children with CAP}

Overall, in the group of 50 children with non-severe CAP, the mean age was $26 \pm 14$ months (median: 24 months, Min/Max: 4/53 months), $18 \%$ of the patients were aged $<1$ year and $50 \%$ were aged $<2$ years. The samples were analyzed using $1 / 400$ and $1 / 1600$ dilutions as these were considered to be the sample dilutions which would yield results on the linear range for all of the used bead sets. Furthermore, by using two distinct dilutions, we could identify cases that were out of the linear range for having either extremely high or low levels of antibodies and, therefore, needed further dilutions. The results from the evaluation of the basal levels of antibodies (in the acute phase of disease) from 50 children with non-severe CAP using the 1/1600 dilution are shown in Fig. 6. The MFI values from the acute phase serum samples varied extensively, ranging from 36 to 1368 for Ply; from 41 to 17,851 for CbpA; from 98 to 6814 for PspA1 and 2; from 36 to 5839 for PcpA; from 47 to 10,526 for PhtD; from 116 to 5853 for SP1732-3; from 28 to 13,645 for SP2216-1; from 38 to 1234 for $H$. influenzae; and from 37 to 472 for $M$. catarrhalis. The frequency of $\geq 1.5$-, 2 -, and 4 -fold increases in the MFI values between the acute and convalescent samples analyzed using both $1 / 400$ and $1 / 1600$ dilutions are shown in Table 2, demonstrating that the assay was able to detect increases in the antibody levels with all bead sets.

\section{Discussion}

In this report we describe the development and careful laboratory validation of a bead based multiplex immunoassay for the simultaneous evaluation of the presence of serum antibodies against $S$. pneumoniae, $H$. influenzae, and $M$. catarrhalis using protein antigens, including several vaccine candidates. Although the use of multiplex assays using pneumococcal proteins has been reported previously (Lebon et al., 2011; Prevaes et al., 2012; Verhaegh et al., 2012), the description of such assays has only been partially published (Shoma et al., 2011), and no data on aspects such as the specificity and comparability with the other assays is available so far. Additionally, this is the first study reporting the validation of a multiplex assay for this specific set of pathogens causing respiratory tract disease, including the information on the possible interference of heterologous inhibition (Prevaes et al., 2012; Verhaegh et al., 2012).

Here we demonstrate that the described assay was specific, reaching $>92 \%$ of homologous inhibition for all coupled beads with only few cases of heterologous inhibition. Cross-reactivity was found between CbpA and PspA1 and 2, which may be explained by the extensive homology described between these proteins (Brooks-Walter et al., 1999). Regarding the occurrence of heterologous inhibition involving the beads conjugated with $H$. influenzae and $M$. catarrhalis antigens, we hypothesize that the conjugation of several proteins in each bead set may have increased the unspecific inhibition. Similarly, when $M$. catarrhalis antigens were added to the sera, the higher total amount of proteins added may have affected the cases of heterologous inhibition by substantially increasing the number of epitopes free in solution for binding with the antibodies against other antigens. Nevertheless, although heterologous inhibition was indeed detected in this test, it is important to recall that the inhibition of binding studies was conducted in experimental conditions that might favor the occurrence of such cases. Firstly, in the inhibition of binding tests, the antigens were added at higher concentrations than the ones used in the conjugation to the beads (except for PcpA). Furthermore, in the inhibition of binding tests the antigens were free in solution, whereas in the usual assay the proteins are permanently linked to the carboxylated surface of the beads (Verkaik et al., 2008) and, therefore, some of their epitopes might be as available to promote heterologous inhibition. Additionally, the tested sera were previously incubated with each antigen for a significantly longer period of time than that of the usual assay ( $12 \mathrm{~h}$ vs. $1 \mathrm{~h}$ ). The similarity between the multiplex and singleplex assays also reinforces the good pattern of specificity demonstrated in the inhibition of binding studies, as the few cases of crossreactivity did not persist when the multiplex assay was performed using the standard protocol. Finally, taking into consideration that previous studies considered an unspecific background as the one with MFI values $>250$ (Kaminski et al., 2013; Verkaik et al., 2008; Waterboer et al., 2006), an importantly low unspecific binding (MFI <20) was found with the uncoupled beads in this assay. Therefore, we conclude that, overall, the developed assay demonstrated good specificity.

Herein, the importance of individually determining the optimal concentration for the conjugation of each antigen was demonstrated. Additionally, when the optimization of the antigen concentration is performed, few concentrations are usually tested (Verkaik et al., 2008). We found that the optimal concentration for the conjugation of most pneumococcal antigens was $1.6 \mu \mathrm{g} / \mathrm{million}$ beads, a value 2.5 -times smaller than the one previously used (Shoma et al., 2011; Verkaik et al., 2008). Nevertheless, an important variation was noticed in the optimal coupling concentration determined for each antigen, reaching a value as high as $32 \mu \mathrm{g} / \mathrm{million}$ beads for the conjugation of PcpA. Importantly, the use of distinct coupling concentrations for each antigen did not affect the fluorescence reading of the assay when the beads were used simultaneously, as demonstrated by the excellent correlation between the singleplex and multiplex assays.

The developed assay demonstrated good robustness, showing good repeatability in intra-assay and day-to-day comparisons. Moreover, the variation between technicians was minimal. Low fluorescence readings provided by any of the used beads exhibited a greater variability when a sample was repeated several times. Nevertheless, even the lowest MFI value strata presented a mean variation $<11 \%$, representing a good level of repeatability. In addition to that, the results obtained when separate preparations of conjugated beads were used were practically identical. Also, although no test for the efficiency of the coupling reaction was performed, we found 
Ply

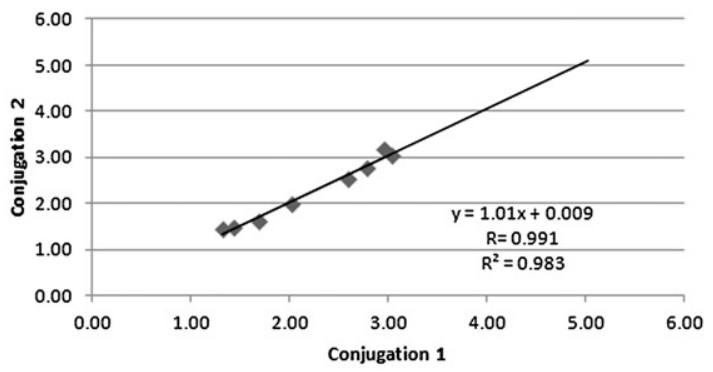

PcpA

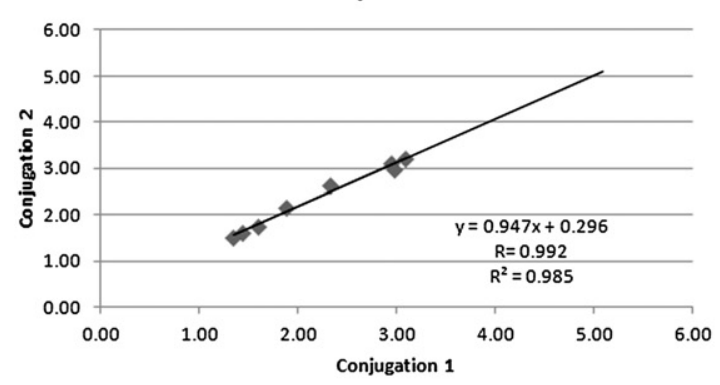

SP2216-1

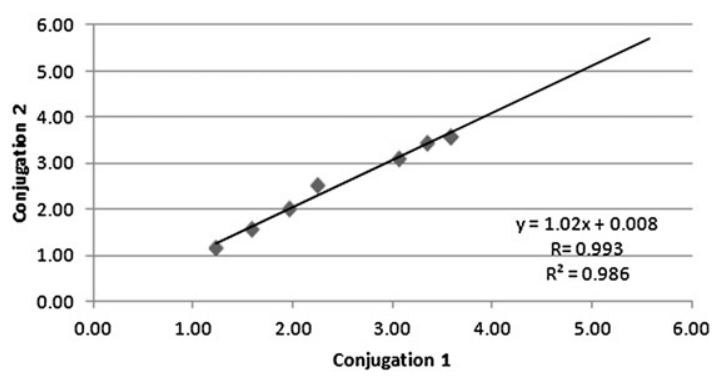

CbpA

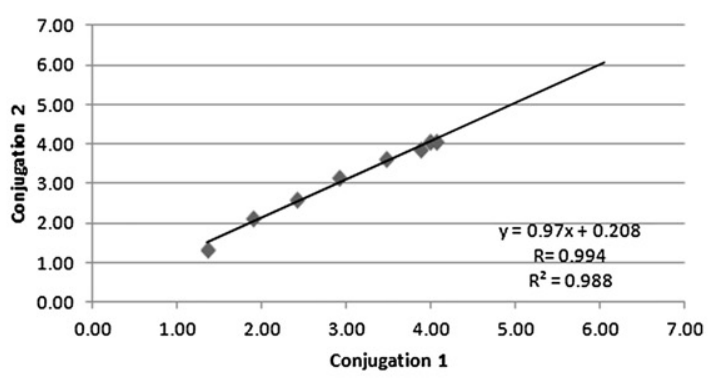

PhtD
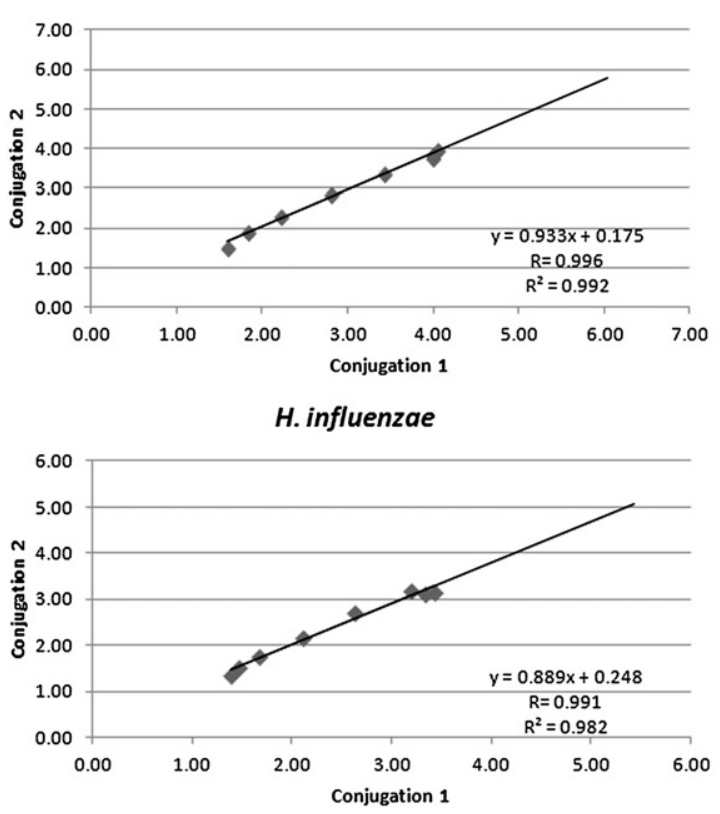

PspA 1 and 2

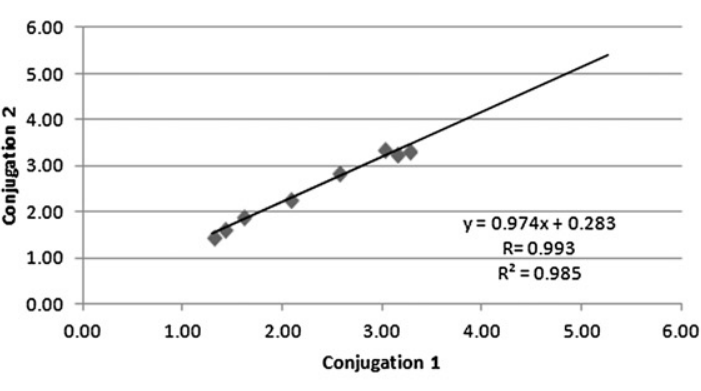

SP1732-3
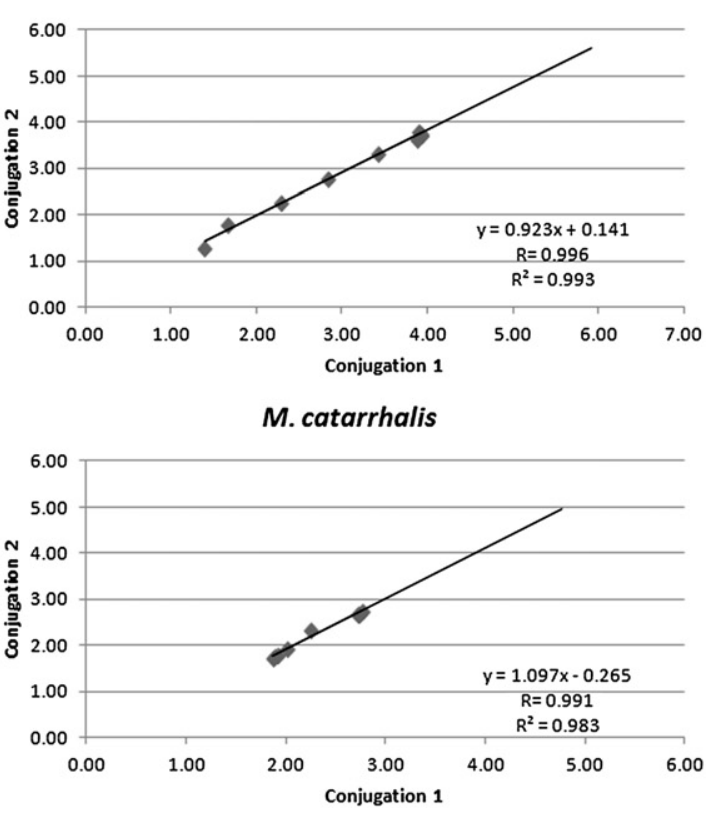

Fig. 5. Comparison of the Mean Fluorescence Intensity (MFI) values, after log transformation, obtained when serial dilutions of Venogamma were assayed using two different batches of conjugated beads. 

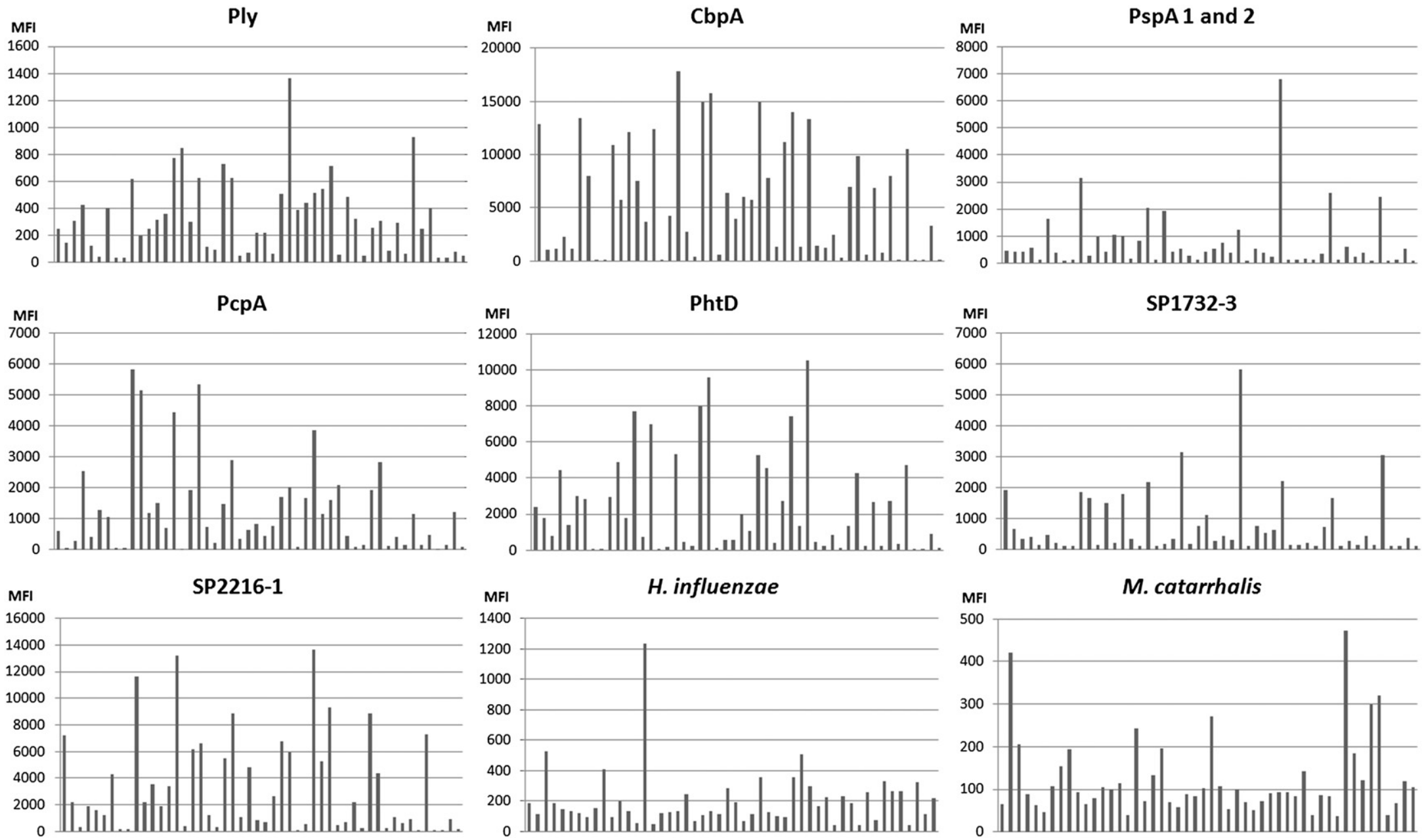

Fig. 6. Results from the FMIA analysis of acute serum samples of 50 children with non-severe CAP using a 1/1600 dilution. Each bar represents the MFI value obtained from 1 serum sample. 
Table 2

Number of $\geq 1.5$-, 2-, and 4-fold increases on the MFI values between the acute and convalescent serum samples of children with non-severe pneumonia, according to the bead-set using $1 / 400$ and 1/1600 dilution.

\begin{tabular}{|c|c|c|c|c|c|c|c|c|c|}
\hline & Ply & CbpA & PspA1 and 2 & PсрA & PhtD & SP1732-3 & SP2216-1 & H. influenzae & M. catarrhalis \\
\hline Increase $\geq 1.5$-fold & 3 & 6 & 3 & 9 & 11 & 4 & 8 & 3 & 2 \\
\hline Increase $\geq 2$-fold & 1 & 4 & 1 & 5 & 4 & 0 & 5 & 3 & 0 \\
\hline Increase $\geq 4$-fold & 0 & 1 & 0 & 0 & 1 & 0 & 1 & 2 & 0 \\
\hline
\end{tabular}

reasonably constant results on separate conjugations of each antigen and tested the coupling reaction with different protein concentrations from which we could indirectly infer the level of protein conjugated to the bead. The assay was linear over a wide dilution range, allowing a reduction of the number of sample dilutions required. Good correlation with ELISA was demonstrated for all tested pneumococcal antigens, including the antigens PspA1 and PspA2 which were analyzed separately by ELISA and conjugated on the same bead set on the FMIA.

Of note, low fluorescence readings and rates of immune response in patient sera were obtained with some bead sets, particularly those conjugated with $H$. influenzae and $M$. catarrhalis antigens. The low rate of response to these proteins might be due to several factors. Firstly, regarding the overall lower values of the fluorescence readings for these antigens, the kinetics of the response to these proteins must be considered when evaluating these data. For instance, for $H$. influenzae Protein D, the levels of IgG are low until the age of 2 years, increasing to moderate levels at 2 to 10 years of age and finally reaching higher levels in adulthood (Akkoyunlu et al., 1996). As half of the tested population was aged $<2$ years, this factor may have contributed to the low levels of IgG found. In addition, for M. catarrhalis antigens, when the serum levels of $\operatorname{IgG}$ against proteins from this pathogen were evaluated, low fluorescence readings were obtained when using pediatric samples (Verhaegh et al., 2012). Finally, the number of previous encounters with these bacteria may influence the basal levels of antibodies against the studied antigens. Therefore, the lower rate of colonization and/or infection by $H$. influenzae or $M$. catarrhalis when compared to S. pneumoniae may contribute to the difference observed between the fluorescence readings yielded by the antigens from each bacterium (Casey et al., 2010). Indeed, the developed assay was able to recognize high levels of antibodies against these agents, e.g. in the sera used to perform the specificity tests, reinforcing that the aforementioned factors may have contributed to the low readings found in some samples.

The evaluation of the paired sera from children with CAP confirmed the existence of a large inter-individual variability in the levels of the analyzed antibodies (Fig. 6), which might reflect previous infection or colonization with S. pneumoniae, $H$. influenzae, and $M$. catarrhalis. This information is valuable because it may be hard to interpret serological tests when dealing with basal levels of antibodies that are on the extremes of the linear range of the assay. In such cases, adjusting the dilution factor of the sample to confirm the result of the assay is recommended. Importantly, children might also respond differently to distinct pneumococcal antigens (Holmlund et al., 2009), as demonstrated in Table 2. All this encourages the use of several antigens when evaluating the immune response to any pathogen. Therefore, the developed assay was shown to be able to quantify varying levels of antibodies with all the coupled bead sets, thereby allowing the detection of increases in the levels of IgG. Indeed, increases in antibodies between acute and convalescent phase samples were found (Table 2), suggesting a recent contact with these pathogens.

Further advantages of this methodology include the use of small volume of sera ( $5 \mu \mathrm{l}$ per duplicate) to screen the samples with several antigens of the studied pathogens. Additionally, the use of protein antigens common to most strains of each of the studied bacteria, respectively, allowed the detection of antibodies against the aforementioned pathogens regardless of the serotype or other variations in the bacterial structures.

Limitations of this study include thus far the absence of a definition of the optimal cut-off point for diagnosis of infection by the studied agents based on testing with true-positive and true-negative samples. This clinical validation step should be accomplished depending on the studied type of infection and its respective gold-standard to guarantee the determination of the best definition of immune response in each case. Further, due to the broad range of antigens used, we could not find a suitable standard for our assay, and therefore the levels of antibodies could not be quantified. Nevertheless, the MFI values are an indirect measure of the levels of antibodies, and several previously published papers used solely this unity to evaluate the immune response to pathogens (Lebon et al., 2011; Prevaes et al., 2012; Shoma et al., 2011; Verhaegh et al., 2012). In this setting the paired samples should always be tested in the same assay. Furthermore, the use of immunoglobulin preparations or human pooled serum has been the routine when dealing with protein antigens (Lebon et al., 2011; Prevaes et al., 2012; Shoma et al., 2011; Verhaegh et al., 2012; Verkaik et al., 2009). In case this methodology would be used in vaccine development, the standard for quantitation of antibody concentrations would be highly recommended.

Due to restrictions in cost, resources, and time, all $H$. influenzae and $M$. catarrhalis antigens were conjugated this time in one bead set per bacteria, and no test was performed with the antigens individually. Consequently, the fluorescence readings from each of those bead sets cannot be attributed to any specific protein. This strategy was also used for PspA1 and 2, and we found a good correlation with ELISA performed for each of these antigens separately, indicating that each protein generates individual contributions to the fluorescence reading of the bead set when multiple antigens are conjugated together. Also, this strategy allowed the simultaneous quantitation of antibodies against several antigens from each agent in a resource-limited setting, 
representing an alternative to increase the sensitivity of the test when individual data for each protein are not required. Comparison with ELISA has been the routine procedure in the validation of FMIA protocols (Kaminski et al., 2013; Lal et al., 2005; Pickering et al., 2002a; Pickering et al., 2002b; van Gageldonk et al., 2008) and the absence of a comparison between the FMIA and ELISA for the antigens from $H$. influenzae and $M$. catarrhalis represents another limitation of our study.

Finally, we present in detail an assay with a set of multiplexed antigens designed for the quantitation of antibodies against common respiratory tract pathogens, which could be easily modified for different studies and different protein antigens. Although there is no currently licensed vaccine using all the studied antigens, most of them are considered vaccine candidates and some are part of experimental vaccines (Adamou et al., 2001; Bologa et al., 2012; Briles et al., 2000; Brooks-Walter et al., 1999; Crain et al., 1990; Croney et al., 2012; Kamtchoua et al., 2013; Khan and Pichichero, 2012; Khan et al., 2012; Poolman et al., 2000; Principi and Esposito, 2011; Saito et al., 2013; Seiberling et al., 2012; Smidt et al., 2013; Tai, 2006). Therefore, this methodology can be used as a basis to create other protocols specific for each of these new potential vaccine combinations.

\section{Conclusion}

We developed an assay for the detection of antibodies against $S$. pneumoniae, $H$. influenzae, and $M$. catarrhalis using small amounts of recombinant proteins which exhibited good patterns of homologous inhibition, had no interference from the multiplexing and was repeatable. Furthermore, concerning the pneumococcal antigens, we also demonstrated that the developed assay was comparable to ELISA. The assay showed a high throughput using discrete volumes of serum samples, representing an important advantage when compared to ELISA, particularly for the testing of pediatric samples. We also showed that the assay detected a wide range of antibody concentrations and increases in paired samples of children with non-severe pneumonia. This assay may be suitable for both etiological studies and for further studies of the immunogenicity of these antigens.

\section{Acknowledgments}

We thank Sanofi-Pasteur for supplying PcpA and PhtD; and Prof Elaine Tuomanen at St. Jude Children's Research Hospital (Memphis, TN) for supplying Ply, CbpA, and PspA1 and Profs Susan Hollingshead and David Briles, and Pat Coan at University of Alabama at Birmingham (Birmingham, AL) for supplying PspA2. We also thank Camilla Virta and Leena Saarinen for their help in the laboratory.

This work was supported by: Bahia State Agency for Research Funding (FAPESB); Brazilian Council for Scientific and Technological Development (CNPq), Brazil, and in part by National Institute for Health and Welfare, Finland. Profs Aldina Barral and Cristiana M. Nascimento-Carvalho are investigators of the CNPq.

Andreas Meinke is an employee of Valneva Austria GmbH.

\section{References}

Adamou, J.E., Heinrichs, J.H., Erwin, A.L., Walsh, W., Gayle, T., Dormitzer, M., Dagan, R., Brewah, Y.A., Barren, P., Lathigra, R., Langermann, S., Koenig, S., Johnson, S., 2001. Identification and characterization of a novel family of pneumococcal proteins that are protective against sepsis. Infect. Immun. 69, 949 (PMID: 11159990).

Akkoyunlu, M., Janson, H., Ruan, M., Forsgren, A., 1996. Biological activity of serum antibodies to a nonacylated form of lipoprotein D of Haemophilus influenzae. Infect. Immun. 64, 4586 (PMID: 8890211).

Bologa, M., Kamtchoua, T., Hopfer, R., Sheng, X., Hicks, B., Bixler, G., Hou, V., Pehlic, V., Yuan, T., Gurunathan, S., 2012. Safety and immunogenicity of pneumococcal protein vaccine candidates: monovalent choline-binding protein A (PcpA) vaccine and bivalent PcpA-pneumococcal histidine triad protein D vaccine. Vaccine 30, 7461 (PMID: 23123106).

Briles, D.E., Hollingshead, S., Brooks-Walter, A., Nabors, G.S., Ferguson, L., Schilling, M., Gravenstein, S., Braun, P., King, J., Swift, A., 2000. The potential to use PspA and other pneumococcal proteins to elicit protection against pneumococcal infection. Vaccine 18, 1707 (PMID: 10689153).

Brooks-Walter, A., Briles, D.E., Hollingshead, S.K., 1999. The $p s p C$ gene of Streptococcus pneumoniae encodes a polymorphic protein, PspC, which elicits cross-reactive antibodies to PspA and provides immunity to pneumococcal bacteremia. Infect. Immun. 67, 6533 (PMID: 10569772).

Casey, J.R., Adlowitz, D.G., Pichichero, M.E., 2010. New patterns in the otopathogens causing acute otitis media six to eight years after introduction of pneumococcal conjugate vaccine. Pediatr. Infect. Dis. J. 29, 304 (PMID: 19935445).

Crain, M.J., Waltman II, W.D., Turner, J.S., Yother, J., Talkington, D.F., McDaniel, L.S., Gray, B.M., Briles, D.E., 1990. Pneumococcal surface protein A (PspA) is serologically highly variable and is expressed by all clinically important capsular serotypes of Streptococcus pneumoniae. Infect. Immun. 58, 3293 (PMID: 1698178).

Croney, C.M., Coats, M.T., Nahm, M.H., Briles, D.E., Crain, M.J., 2012. PspA family distribution, unlike capsular serotype, remained unaltered following introduction of the heptavalent-pneumococcal conjugate vaccine. Clin. Vaccine Immunol. 19, 891 (PMID: 22539473).

De Wals, P., Erickson, L., Poirier, B., Pépin, J., Pichichero, M.E., 2009. How to compare the efficacy of conjugate vaccines to prevent acute otitis media? Vaccine 27, 2877 (PMID: 19366579).

Giefing, C., Meinke, A.L., Hanner, M., Henics, T., Bui, M.D., Gelbmann, D., Lundberg, U., Senn, B.M., Schunn, M., Habel, A., Henriques-Normark, B., Ortqvist, A., Kalin, M., von Gabain, A., Nagy, E., 2008. Discovery of a novel class of highly conserved vaccine antigens using genomic scale antigenic fingerprinting of pneumococcus with human antibodies. J. Exp. Med. 205, 117.

Holmlund, E., Quiambao, B., Ollgren, J., Jaakkola, T., Neyt, C., Poolman, J., Nohynek, H., Käyhty, H., 2009. Antibodies to pneumococcal proteins PhtD, CbpA, and LytC in Filipino pregnant women and their infants in relation to pneumococcal carriage. Clin. Vaccine Immunol. 16, 916.

Kaminski, R.W., Clarkson, K., Kordis, A.A., Oaks, E.V., 2013. Multiplexed immunoassay to assess Shigella-specific antibody responses. J. Immunol. Methods 393, 18 (PMID: 23583496).

Kamtchoua, T., Bologa, M., Hopfer, R., Neveu, D., Hu, B., Sheng, X., Corde, N., Pouzet, C., Zimmermann, G., Gurunathan, S., 2013. Safety and immunogenicity of the pneumococcal pneumolysin derivative PlyD1 in a singleantigen protein vaccine candidate in adults. Vaccine 31, 327 (PMID: 23153437)

Khan, M.N., Pichichero, M.E., 2012b. Vaccine candidates PhtD and PhtE of Streptococcus pneumoniae are adhesins that elicit functional antibodies in humans. Vaccine 30, 2900 (PMID: 22349524).

Khan, M.N., Sharma, S.K., Filkins, L.M., Pichichero, M.E., 2012a. PcpA of Streptococcus pneumoniae mediates adherence to nasopharyngeal and lung epithelial cells and elicits functional antibodies in humans. Microbes Infect. 14, 1102 (PMID: 22796387).

Kilpi, T., Herva, E., Kaijalainen, T., Syrjänen, R., Takala, A.K., 2001. Bacteriology of acute otitis media in a cohort of Finnish children followed for the first two years of life. Pediatr. Infect. Dis. J. 20, 654 (PMID: 11465836)

Korppi, M., Leinonen, M., Ruuskanen, O., 2008. Pneumococcal serology in children's respiratory infections. Eur. J. Clin. Microbiol. Infect. Dis. 27, 167 (PMID: 18087733).

Lal, G., Balmer, P., Stanford, E., Martin, S., Warrington, R., Borrow, R., 2005. Development and validation of a nonaplex assay for the simultaneous quantitation of antibodies to nine Streptococcus pneumoniae serotypes. J. Immunol. Methods 296, 135 (PMID: 15680158).

Lebon, A., Verkaik, N.J., Labout, J.A., de Vogel, C.P., Hooijkaas, H., Verbrugh, H.A., van Wamel, W.J., Jaddoe, V.W., Hofman, A., Hermans, P.W., Ma, J., Mitchell, T.J., Moll, H.A., van Belkum, A., 2011. Natural antibodies against 
several pneumococcal virulence proteins in children during the prepneumococcal-vaccine era: the generation R study. Infect. Immun. 79, 1680 (PMID: 21282409).

McDaniel, L.S., Ralph, B.A., McDaniel, D.O., Briles, D.E., 1994. Localization of protection-eliciting epitopes on PspA of Streptococcus pneumoniae between amino acid residues 192 and 260. Microb. Pathog. 17, 323 (PMID: 7723659).

Murphy, T.F., Parameswaran, G.I., 2009. Moraxella catarrhalis, a human respiratory tract pathogen. Clin. Infect. Dis. 49, 124 (PMID: 19480579).

Ogunniyi, A.D., Woodrow, M.C., Poolman, J.T., Paton, J.C., 2001. Protection against Streptococcus pneumoniae elicited by immunization with pneumolysin and CbpA. Infect. Immun. 69, 5997 (PMID: 11553536).

Orihuela, C.J., Mahdavi, J., Thornton, J., Mann, B., Wooldridge, K.G., Abouseada, N., Oldfield, N.J., Self, T., Ala'Aldeen, D.A., Tuomanen, E.I., 2009. Laminin receptor initiates bacterial contact with the blood brain barrier in experimental meningitis models. J. Clin. Invest. 119, 1638 (PMID:19436113).

Pickering, J.W., Martins, T.B., Greer, R.W., Schroder, M.C., Astill, M.E., Litwin, C.M., Hildreth, S.W., Hill, H.R., 2002a. A multiplexed fluorescent microsphere immunoassay for antibodies to pneumococcal capsular polysaccharides. Am. J. Clin. Pathol. 117, 589 (PMID: 11939734).

Pickering, J.W., Martins, T.B., Schroder, M.C., Hill, H.R., 2002b. Comparison of a multiplex flow cytometric assay with enzyme-linked immunosorbent assay for quantitation of antibodies to tetanus, diphtheria, and Haemophilus influenzae type b. Clin. Diagn. Lab. Immunol. 9, 872 (PMID: 12093688).

Poolman, J.T., Bakaletz, L., Cripps, A., Denoel, P.A., Forsgren, A., Kyd, J., Lobet, Y., 2000. Developing a nontypeable Haemophilus influenzae (NTHi) vaccine. Vaccine 19, 108 (PMID: 11163473).

Posfay-Barbe, K.M., Galetto-Lacour, A., Grillet, S., Ochs, M.M., Brookes, R.H., Kraehenbuhl, J.D., Cevey-Macherel, M., Gehri, M., Gervaix, A., Siegrist, C.A., 2011. Immunity to pneumococcal surface proteins in children with community-acquired pneumonia: a distinct pattern of responses to pneumococcal choline-binding protein A. Clin. Microbiol. Infect. 17, 1232 (PMID: 21040158).

Prevaes, S.M., van Wamel, W.J., de Vogel, C.P., Veenhoven, R.H., van Gils, E.J., van Belkum, A., Sanders, E.A., Bogaert, D., 2012. Nasopharyngeal colonization elicits antibody responses to staphylococcal and pneumococcal proteins that are not associated with a reduced risk of subsequent carriage. Infect. Immun. 80, 2186 (PMID: 22451514).

Principi, N., Esposito, S., 2011. Universal protein vaccines against Neisseria meningitidis serogroup B, Streptococcus pneumoniae and influenza. Hum. Vaccin. 7, 905 (PMID: 21865879).

Rodgers, G.L., Klugman, K.P., 2011. The future of pneumococcal disease prevention. Vaccine 29, 43 (PMID: 21896352).

Rudan, I., Boschi-Pinto, C., Biloglav, Z., Mulholland, K., Campbell, H., 2008. Epidemiology and etiology of childhood pneumonia. Bull. World Health Organ. 86, 408 (PMID: 18545744).

Saito, R., Matsuoka, S., Fujinami, Y., Nonaka, S., Ichinose, S., Kubota, T., Okamura, N., 2013. Role of Moraxella catarrhalis outer membrane protein CD in bacterial cell morphology and autoaggregation. Res. Microbiol. 164, 236 (PMID: 23257176).
Seiberling, M., Bologa, M., Brookes, R., Ochs, M., Go, K., Neveu, D., Kamtchoua, T., Lashley, P., Yuan, T., Gurunathan, S., 2012. Safety and immunogenicity of a pneumococcal histidine triad protein $\mathrm{D}$ vaccine candidate in adults. Vaccine 30, 7455 (PMID: 23131206).

Shaper, M., Hollingshead, S.K., Benjamin Jr., W.H., Briles, D.E., 2004. PspA protects Streptococcus pneumoniae from killing by apolactoferrin, and antibody to PspA enhances killing of pneumococci by apolactoferrin [corrected]. Infect. Immun. 72, 5031 (PMID: 15321996).

Shoma, S., Verkaik, N.J., de Vogel, C.P., Hermans, P.W., van Selm, S., Mitchell, T.J., van Roosmalen, M., Hossain, S., Rahman, M., Endtz, H.P., van Wamel, W.J., van Belkum, A., 2011. Development of a multiplexed bead-based immunoassay for the simultaneous detection of antibodies to 17 pneumococcal proteins. Eur. J. Clin. Microbiol. Infect. Dis. 30, 521 (PMID: 21086008)

Simell, B., Lahdenkari, M., Reunanen, A., Käyhty, H., Väkeväinen, M., 2008. Effects of ageing and gender on naturally acquired antibodies to pneumococcal capsular polysaccharides and virulence-associated proteins. Clin. Vaccine Immunol. 15, 1391 (PMID: 18596205).

Smidt, M., Bättig, P., Verhaegh, S.J., Niebisch, A., Hanner, M., Selak, S., Schüler W., Morfeldt, E., Hellberg, C., Nagy, E., Lundberg, U., Hays, J.P., Meinke, A., Henriques-Normark, B., 2013. Comprehensive antigen screening identifies Moraxella catarrhalis proteins that induce protection in a mouse pulmonary clearance model. PLoS One 8, e64422 (PMID: 23671716).

Tai, S.S., 2006. Streptococcus pneumoniae protein vaccine candidates: properties, activities and animal studies. Crit. Rev. Microbiol. 32, 139 (PMID: 16893751).

van der Poll, T., Opal, S.M., 2009. Pathogenesis, treatment, and prevention of pneumococcal pneumonia. Lancet 374, 1543 (PMID: 19880020).

van Gageldonk, P.G., van Schaijk, F.G., van der Klis, F.R., Berbers, G.A., 2008. Development and validation of a multiplex immunoassay for the simultaneous determination of serum antibodies to Bordetella pertussis, diphtheria and tetanus. J. Immunol. Methods 335, 79 (PMID: 18407287).

Verhaegh, S.J., Stol, K., de Vogel, C.P., Riesbeck, K., Lafontaine, E.R., Murphy, T.F., van Belkum, A., Hermans, P.W., Hays, J.P., 2012. Comparative analysis of the humoral immune response to Moraxella catarrhalis and Streptococcus pneumoniae surface antigens in children suffering from recurrent acute otitis media and chronic otitis media with effusion. Clin. Vaccine Immunol. 19, 914 (PMID: 22539468).

Verkaik, N., Brouwer, E., Hooijkaas, H., van Belkum, A., van Wamel, W., 2008. Comparison of carboxylated and Penta-His microspheres for semiquantitative measurement of antibody responses to His-tagged proteins. J. Immunol. Methods 335, 121 (PMID: 18417151).

Verkaik, N.J., de Vogel, C.P., Boelens, H.A., Grumann, D., Hoogenboezem, T., Vink, C., Hooijkaas, H., Foster, T.J., Verbrugh, H.A., van Belkum, A., van Wamel, W.J., 2009. Anti-staphylococcal humoral immune response in persistent nasal carriers and noncarriers of Staphylococcus aureus. J. Infect. Dis. 199, 625 (PMID: 19199541).

Waterboer, T., Sehr, P., Pawlita, M., 2006. Suppression of non-specific binding in serological Luminex assays. J. Immunol. Methods 309, 200 (PMID: 16406059). 